\title{
Book Review: Urbane Konflikte und die Krise der Demokratie. Stadtentwicklung, Rechtsruck und Soziale Bewegungen
}

\author{
Daniel Mullis \\ Leibniz-Institut Hessische Stiftung Friedens- und Konfliktforschung, Frankfurt am Main, Deutschland \\ Correspondence: Daniel Mullis (mullis@hsfk.de)
}

Published: 20 January 2022

Bescherer, P., Burkhardt, A., Feustel, R., Mackenroth, G., und Sievi, L.: Urbane Konflikte und die Krise der Demokratie: Stadtentwicklung, Rechtsruck und Soziale Bewegungen, Verlag Westfälisches Dampfboot, Münster, 246ff., ISBN 978-389691-057-8, EUR 28,00, 2021.

Großstädte werden gemeinhin als Orte kosmopoliter Milieus und multikultureller Lebensstile, des Fortschrittes, der gesellschaftlichen Inklusion und der demokratischen Teilhabe gedeutet. Die neue Wohnungsfrage, neoliberale Restrukturierungen sowie Austeritätspolitiken lassen Wissenschaftler:innen hingegen schon länger von der ,Krise der Städte" (Heitmeyer et al., 1998) sprechen und deren integratives Potential in Frage stellen. Seit der zweiten Hälfte der 2000er Jahre werden Prozesse der sozialen Exklusion gerade von linken sozialen Bewegungen konfrontiert. Während letzteres das Bild der pluralistischen Stadt noch hochzuhalten erlaubt, zeigt sich zunehmend, dass in Städten auch rechtsextreme Tendenzen erstarken. Zwar schnitt die Alternative für Deutschland (AfD) in deutschen Großstädten mit über 250000 Einwohner:innen bei der Bundestagswahl 2017, als sie zum ersten Mal mit 12,6\% in den Bundestag einzog, lediglich in den beiden sächsischen Metropolen Leipzig (18,3\%) und Dresden (22,2\%) besser ab als auf Bundesebene (Bernet et al., 2019:13), gleichzeitig kaschiert der vereinheitlichende Blick auf kommunale Wahlergebnisse die bisweilen starken Resultate der Partei in manch Stadtteilen der Republik. Hinzu kommt, dass gerade Kleinstädte, besonders akzentuiert in Ostdeutschland, schon länger als zentrale Orte rechtsextremer Politik und Bewegung beschrieben werden (Heitmeyer, 2018:324).

Hier setzt der Band „Urbane Konflikte und die Krise der Demokratie“ von Peter Bescherer, Anne Burkhardt, Robert Feustel, Gisela Mackenroth und Luzia Sievi, erschie- nen 2021 im Verlag Westfälisches Dampfboot, an. Die Autor:innen legen eine der ersten qualitativen Studien zum aktuellen Rechtsextremismus in deutschen Großstädten vor. Untersucht werden ,,verschiedene städtische Problemlagen daraufhin, ob sie Unrechtsempfindungen und Bedrohungsgefühle hervorrufen, die im System der repräsentativen Demokratie keinen adäquaten Ausdruck finden“" (Bescherer et al., 2021:22) und damit von rechts politisiert werden können. Es handelt sich um die Abschlusspublikation des von 2017 bis 2020 vom Bundesministerium für Bildung und Forschung (BMBF) geförderten Projektes Populismus und Demokratie in der Stadt (Podesta). Empirisch werden „Mikrokonflikte“ (Bescherer et al., 2021:31) um die Wohnungsfrage, Verkehrs- und Energiewende sowie um städtebauliche Projekte in Leipzig und Stuttgart untersucht. Leitend ist der Anspruch, die Befunde zwischen den Städten zu kontrastieren. Trotz der gemeinsamen Autor:innenschaft sind die jeweiligen Kapitel von einzelnen Personen gezeichnet, was deren Eigenständigkeit unterstreicht.

In der Einleitung von Bescherer und Mackenroth werden die zentralen Konzepte geklärt. Anschließend führen Burkhardt und Feustel in antiurbane Narrative der kontemporären extremen Rechten mit einem Fokus auf Positionen der AfD ein. Bevor es zur Darstellung der Konflikte in den beiden Städten geht, werden von Bescherer und anschließend Feustel Einschätzungen aus Gesprächen mit Expert:innen vorgestellt. Dann folgen die Fallstudien: Die erste widmet sich dem Konflikt um einen Garagenhof in Leipzig (Bescherer und Feustel); die zweite schildert die Entwicklung einer deutschen Gelbwestenbewegung in Stuttgart, die gegen Dieselfahrverbote mobilisierte (Burkhardt und Sievi); die dritte wendet sich Konflikten um Wohnraum in Stuttgart zu (Mackenroth); und die vierte thematisiert abschließend solida- 
rische Perspektiven gegen die extreme Rechte in mietenpolitischen Auseinandersetzungen in Leipzig (Bescherer). Die letzte Fallstudie fällt im Gefüge des Buches etwas aus dem Kontext. Anders als in den anderen Studien wird hier explizit positiv Partei ergriffen und mittels eines aktivistischen Ansatzes geforscht. Die Studie vermag die konkreten Spannungsverhältnisse aufzuzeigen, in denen die einzelnen Subjekte vor Ort sich bewegen und legt dar, dass rechtsextreme Einstellungsmuster bei Mieter:innen nicht geschlossen und demokratisch einzuholen sind. Dennoch wirkt sie im Gesamtgefüge des Bandes etwas isoliert.

Konzeptuell leitend ist das im Anschluss an Dubiel formulierte Konzept der „populistischen Lücke“ (Bescherer et al., 2021:20-25). Diese entstehe dadurch, dass etablierte Parteien soziale Konflikte nicht mehr adressierten und spezifische Milieus nicht mehr repräsentierten. Die Lücke werde dann von populistischen Agiteur:innen bearbeitet und die Subjekte durch die extreme Rechte politisch reintegriert. Die Autor:innen sind sich der Grenzen des Ansatzes bewusst. So sei zum einen problematisch, dass erfolgreiche rechtspopulistische Ansprache in der Tendenz zu einer Funktion der Abwesenheit einer adressierenden Politik durch nichtpopulistische Parteien werde und andererseits die aktive und eigenständige Kraft rechtsextremer Inhalte keine Beachtung fände. Zudem schaffe auch ,funktionierende Politik“ rechtsextreme Anschlüsse, was etwa Natascha Strobl (2021) jüngst deutlich herausgearbeitet hat, weshalb die These der Dysfunktionalität von vornherein zu kurz greife. Die Autor:innen entscheiden sich dennoch für das Konzept, zumal es erlaube, den Erfahrungen und damit verbundenen politischen Begründungsnarrativen für die Zuwendung zur extremen Rechten nachzugehen.

Die Fokussierung auf die populistische Lücke entspricht dem Debattenstand Mitte der 2010er Jahre zum Aufstieg der AfD. Geprägt von Arbeiten wie „Abstiegsgesellschaft“ (Nachtwey, 2016) oder „Rückkehr nach Reims“ (Eribon, 2016 [2009]) wurde intensiv über ,objektive“ Gründe für die Erfolge diskutiert. Es ging um Modernisierungsverlierer:innen, Abgehängte, Prekarisierte, um Menschen, die das Gefühl hätten, in der Warteschlange zu stehen, und dabei von Migrant:innen und Geflüchteten übervorteilt würden (vgl. Hochschild, 2017). Vor dem Hintergrund anhaltender Erfolge der AfD, der Akzentuierung der Polarisierung zwischen Ost- und Westdeutschland, der Attentate von u.a. Halle und Hanau sowie der Proteste eines diffusen rechts-offenen bis rechtsextremen Spektrums von CoronaPandemie-Leugner:innen veränderte sich die Perspektivensetzung - wie die Autor:innen selbst schreiben (Bescherer et al., 2021:97): Verstärkt rücken tradierte lokale Verhältnisse und politische Kulturen, rassistische Kontinuitäten und Realitäten, irrationale Verschwörungsideologien sowie die Rolle von sozialen Medien in den Fokus. Das heißt nicht, dass ,objektivierbare" soziale Verhältnisse, Lebenslagen und Desintegration keine Rolle spielten, deutlich wurde aber, dass die rechtsextremen Entwicklungen nicht hinreichend durch sie erklärt werden können.

In diese Richtung deuten sodann die Befunde der Studien selbst. Die Autor:innen können zwar städtische ,populistische Lücken“ darlegen, eine aktive Politisierung von städtischen Themen durch rechtspopulistische Akteur:innen können sie jedoch kaum aufzeigen (Bescherer et al., 2021:210220). Detailliert arbeiten sie heraus, dass die Stadt für die extreme Rechte „Kampfplatz, Projektionsfläche und Sehnsuchtsort" (Bescherer et al., 2021:36) zugleich ist. Rechte Stadtdiskurse kreisten im Wesentlichen um „Sicherheit und Verkehr“, „Wohnen und Miete“, „Architektur und Städtebau“ sowie „Flucht und Migration" (Bescherer et al., 2021:43). Dabei werde das Narrativ des bedrohten deutschen Volkes bedient und, ,auf den spezifischen städtischen Kontext umgemünzt" (Bescherer et al., 2021:57). Es gelte die kosmopolite Stadt zurückzuerobern. Gleichzeitig erkennen die Autor:innen kein „genuines Interesse“ der extremen Rechten an Stadt. Auch die befragten Expert:innen wollen ,rechte Deutungs- und Handlungsmuster“ nicht als ,städtisches Phänomen betrachten" (Bescherer et al., 2021:68), zumal die AfD keine Stadtpolitik mache. Explizit werde seitens der befragten Expert:innen, die angesichts des Rechtsrucks ratlos wirkten, betont, dass die extreme Rechte städtische Lücken der Repräsentation nicht bewirtschafte (Bescherer et al., 2021:91). Auf der Suche nach Ursachen folge seitens der Expert:innen vielfach der Verweis ,auf die mediale Ebene, die einen wachsenden Einfluss ausübe und bisweilen die realen Gegebenheiten vor Ort auffällig verzerre" (Bescherer et al., 2021:88). Gleichzeitig werde die Notwendigkeit, Sozialpolitik auszuweiten, Menschen wieder demokratisch zu inkludieren und Urbanität vor Ort zu stärken, als lokale Desiderate formuliert (Bescherer et al., 2021:71). Bilanzierend wird in Abgrenzung zu den Interviews betont, dass , die vollständige Zurückweisung städtischer Ursachen des Rechtspopulismus ebenso wenig plausibel [ist] wie ihre vollständige Lokalisierung“" (Bescherer et al., 2021:77).

Letzteres zeigen sie in den konkreten Fallstudien differenziert und empirisch solide. Im ,exemplarischen“ Konflikt um einen Garagenhof in Leipzig, der einem Schulcampus weichen soll, wird die Auseinandersetzung um ein städtebauliches Projekt aufgearbeitet. Die Garagen seien mehr als Parkplätze. Seit Jahrzehnten dienten sie als sozialer Treffpunkt, dementsprechend versuchten die Nutzer:innen sie zu erhalten (Bescherer et al., 2021:98). Auf der Suche nach Unterstützung hätte sich die Garagengemeinschaft an politische Parteien gewandt, wobei einzig die AfD sich der Sache wirklich angenommen hätte (Bescherer et al., 2021:113). Was auf den ersten Blick wie das Bedienen der Lücke aussieht, erweist sich, so die Autor:innen, als komplexer: Die Garagengemeinschaft hätte die Dienste der AfD im Parlament in Anspruch genommen, darüber hinaus aber ein eher instrumentelles und ideologisch distanziertes Verhältnis behalten (Bescherer et al., 2021:118). 
Auch in Stuttgart ging es beim Protest der Gelbwesten um Autos. Hier war es ein Diesel-Fahrverbot, das die Bewegung entfachte. Deutlich wird hier, so die Autor:innen, dass gerade „Umwelt- und Gesundheitsschutz“ ein erhebliches Mobilisierungspotential für die extreme Rechte berge, was angesichts der Corona-Pandemie sowie des ökologischen Umbaus in der Zukunft ein Mehr an Konflikten erwarten lasse. Im Zentrum stünden auch hier Fragen von tradierten Identitäten und Status, die von den ,grünen ' Politiken bedroht würden, welche die eigenen ,Freiheiten " und ,Rechte ' beschnitten (Bescherer et al., 2021:149). Es ist gerade das populistische Narrativ ,wir hier unten gegen die da oben", das verfange (Bescherer et al., 2021:152). Anders gelagert ist die Situation im Stuttgarter Stadtteil Hallschlag. In dem marginalisierten Viertel hat eine Wohnungsbaugesellschaft saniert und nachverdichtet, was zu Ängsten vor Verdrängung geführt habe. Gleichzeitig erreichte die AfD für Stuttgarter Verhältnisse 2017 gute Resultate von rund 15 Prozent. Diese Erfolge seien aber weniger durch die aktive Bearbeitung einer Lücke zustande gekommen als dadurch, dass im Viertel rassistische Ressentiments und antidemokratische Einstellungen präsent seien, die durch die urbane Transformation potenziert worden seien. Konflikte um bezahlbaren Wohnraum sowie die Nutzung des öffentlichen Raums würden als „ethnisierte Ressourcenkonflikte“ gedeutet (Bescherer et al., 2021:167).

Bescherer, Burkhardt, Feustel, Mackenroth und Sievi leisten mit ihrer qualitativen Studie in Leipzig und Stuttgart insgesamt einen wichtigen Beitrag zum Verständnis urbaner Konfliktlinien und dem Erstarken der extremen Rechten. Insgesamt hätte jedoch die wechselseitige Kontrastierung der Fallstudien stärker ausfallen können. Deutlich wird an dieser Stelle auch, dass es dem Band gutgetan hätte, wenn der noch junge, aber durchaus vorhandene Forschungsstand zu Rechtsextremismus im urbanen Kontext auch mit Blick auf internationale Arbeiten konsequenter aufgearbeitet worden wäre. (Für die deutschsprachige Debatte vgl. Mullis und Miggelbrink, 2021:193 f.; international gibt es insb. Arbeiten zum Aufstieg von Trump und dem Brexit, die die urbane Frage thematisieren.) Dies hätte die Möglichkeit geschaffen, die lokalen Befunde stärker an übergeordnete Narrative zurückzubinden und auch gemeinsame Trends klarer zu benennen. Der Qualität der Beiträge selbst tut dies jedoch keinen Abbruch.
Haftungsausschluss. Publisher's note: Copernicus Publications remains neutral with regard to jurisdictional claims in published maps and institutional affiliations.

\section{Literatur}

Bernet, T., Bescherer, P., Beurskens, K., Feustel, R., und Michel, B.: Stadt von Rechts?, sub \urban, 7, 7-22, 2019.

Bescherer, P., Burkardt, A., Feustel, R., Mackenroth, G., und Sievi, L.: Urbane Konflikte und die Krise der Demokratie: Stadtentwicklung, Rechtsruck und Soziale Bewegungen, Westfälisches Dampfboot, Münster, ISBN 978-3-89691-057-8, 2021.

Eribon, D.: Rückkehr nach Reims, Suhrkamp, Berlin, ISBN 978-3518-07252-3, 2016 [2009].

Heitmeyer, W.: Autoritäre Versuchungen, Suhrkamp, Berlin, ISBN 978-3-518-12717-9, 2018.

Heitmeyer, W., Dollase, R., und Backes, O.: Die Krise der Städte, Suhrkamp, Frankfurt am Main, ISBN 978-3-518-12036-1, 1998.

Hochschild, A. R.: Fremd in ihrem Land, Campus, Frankfurt, ISBN 978-3-593-50766-8, 2017.

Mullis, D. und Miggelbrink, J.: Rechtsextremismusforschung in der deutschsprachigen Humangeographie, Geogr. Z., 4/21, 184-207, https://doi.org/10.25162/gz-2021-0016, 2021.

Nachtwey, O.: Die Abstiegsgesellschaft, Suhrkamp, Berlin, ISBN 978-3-518-12682-0, 2016.

Strobl, N.: Radikalisierter Konservatismus, Suhrkamp, Berlin, ISBN 978-3-518-12782-7, 2021. 